CA125 with the tumor burden evaluated through the volumetric parameters of FDG-PET/CT FDG in patients with advanced epithelial ovarian cancer (EOC) before primary treatment.

Methodology Sixty-six patients with advanced stage high grade serous ovarian carcinoma (HGSOC) or undifferentiated carcinoma (UOC) were included. Serum HE4, serum CA125 and FDG PET/CT were performed before primary treatment. Volumes of interest (VOIs) were delimited on every pathological uptake in PET images. Whole-body metabolic tumor volume (wbMTV) and total lesion glycolysis (wbTLG) were calculated as the sum of every single VOI value. SUVmax thresholds were set at $40 \%$ and 50\%. Four VOIs subgroups were defined for analysis: carcinomatosis, retroperitoneal, supradiaphragmatic and metastases. MTV and TLG values were calculated for each of them. The associations between these parameters and serum tumor markers were assessed through Pearson and Spearman tests.

Result(s)* When correlating wbMTV and wbTLG with both CA125 and HE4, significant associations were found. The strongest correlation was observed between HE4 and wbMTV40\% $\quad(\mathrm{r}=0.61, \mathrm{p}<0.001)$. Peritoneal carcinomatosis MTV exhibited a statistically significant correlation with both tumor markers. Pearson's correlation coefficient was 0.61 $(p<0.0001)$ between HE4 and MTV40\% and 0,29 $(p=0.02)$ between CA125 and MTV40\%. Neither the retroperitoneal nor the supradiaphragmatic or metastatic disease assessed by MTV and TLG showed any correlation with these tumor markers.

Conclusion* Peritoneal tumor burden measured by FDG PET/ CT volumetric parameters correlates better with HE4 than with CA125 in patients with advanced epithelial ovarian cancer. These results support the increasing utility of HE4 to improve the stratification of these patients in clinical practice

\section{PARP INHIBITORS IN REAL-WORLD PRACTICE FOR NEWLY DIAGNOSED OVARIAN CANCER: THE EFFECT OF ONLINE EDUCATION ON CLINICIAN COMPETENCE AND CONFIDENCE}

${ }^{1} \mathrm{G}$ Fisher*, 'A Furedy, '1 Vandenbroucque, ${ }^{2} \mathrm{~J}$ Ledermann. ${ }^{1}$ Medscape Global Education, London, UK; ${ }^{2}$ University College London Cancer Institute, London, UK

\subsection{6/ijgc-2021-ESGO.463}

Introduction/Background* This study determined whether online continuing medical education (CME) could improve the competence of obstetricians/gynaecologists (obs/gyns) and oncologists (oncs) regarding the selection and application of PARP inhibitor maintenance strategies for patients with newly diagnosed advanced ovarian cancer

Methodology A 30-minute online video discussion between four expert faculty was launched for physicians outside the USA December 2020 with data collected to May 2021. Educational effect assessed with repeated-pairs pre-/post-activityindividual participants serving as their own control. 3 multiple-choice, knowledge questions and 1 self-efficacy, 5-point Likert scale confidence question were analysed. McNemar's test assessed pre- to post-activity change $(5 \%$ significance level, $P<.05)$. Magnitude of change in total number of correct responses overall, and for each question, determined with Cohen's d (<.2=Modest, .20-.49=Small, .50-.79=Moderate, $>.80=$ Large.
Result(s)* 216 obs/gyns and 80 oncs completed pre- and postactivity questions. Positive educational effect was observed for obs/gyns (small effect, Cohen's $\mathrm{d}=.34, \mathrm{P}<.001$; average\% of correct responses increasing from 46 to 57\%) and oncs (moderate effect, Cohen's $\mathrm{d}=.51, \mathrm{P}<.001$; average $\%$ of correct responses increasing from 60 to $73 \%$ ). Increases in correct responses post-activity were seen for questions on selection of the right diagnostic testing (\% relative improvement, obs/gyn: $21 \%$; oncs $15 \%$ ), selection of treatment/maintenance strategy (obs/gyns: 10\%, oncs: 4\%), and management of myelosuppression (obs/gyns: 37\%; oncs: 44\%). The \% of participants answering all questions correctly increased from 12 to $25 \%$ for obs/gyns and from 23 to $43 \%$ for oncs. Pre-activity competence was low for all questions and despite improvements, this remained modest post-activity. Confidence selecting an appropriate PARP inhibitor maintenance strategy for patients with newly diagnosed ovarian cancer increased post-activity with $47 \%$ of obs/gyn and $44 \%$ of oncs with improved confidence. Overall, $41 \%$ of obs/gyns and $41 \%$ of oncs improved their competence by answering at least one more question correctly post-activity.

Conclusion* This on-demand, online video panel discussion had a positive educational impact. However, significant education gaps were evident pre-activity that remained post-activity in a high proportion of participants. There is a need for further education to increase the competence and confidence of clinicians in application of PARP inhibitor maintenance regimens in real world practice

\section{THE TRANS-RETRO-PERITONEAL SINGLE-PORT ENDOSCOPIC ACCESS FOR COMPREHENSIVE STAGING OF EARLY OVARIAN CANCER: CASE REPORT AND VIDEO}

L Lelievre*, P Mathevet. Lausanne University Hospital, Lausanne, Switzerland

\subsection{6/ijgc-2021-ESG0.464}

Introduction/Background* We developed a new mini-invasive access to perform an endoscopic extra-peritoneal para-aortic lymphadenectomy via a single-port umbilical device. This technique allows, per se, access to the peritoneal space, so complementary staging procedures can be performed via the same single-port. Here we describe the use of the technique to stage an early ovarian cancer patient, including a pelvic and para-aortic lymphadenectomy up to the left renal vein. So far in gynaecologic surgery, two distinct types of endoscopic accesses have been used to perform paraaortic lymphadenectomies: trans-peritoneal and extra-peritoneal, each with advantages and disadvantages. We previously reported the use of our technique to stage locally advanced cervical cancer patients prior to concomitant radio-chemotherapy, combining the advantages of those two approaches while eliminating their disadvantages. Here we report the use of this technique to perform a complete staging for an ovarian cancer patient. Methodology This innovative approach combines both an extra-peritoneal and an intra-peritoneal procedure via the same umbilical incision using one single trocar (Applied Medical Gelpoint $\mathrm{Mini}^{\circ}$ ) The key point is to grab the peritoneum overlying the aorta bifurcation, raise it to the umbilicus and re-introduce the single-port device into the retroperitoneal space. All the paraaortic lymphatic basins are dissected up to the left renal vein. Once the para-aortic lymphadenectomy is 
done, the trocar is reinserted into the peritoneal space, always through the same incision, to perform the endoscopic lymphadenectomy and bilateral pelvic lymphadenectomy, along with the hysterectomy. The procedure was undertaken for a 66 years old patient, with a supposed FIGO IC2 ovarian tumour previously diagnosed after a trans-umbilical single-port endoscopic bilateral adnexectomy.

Result(s)* The intervention has been successful in this patient with FIGO stage IC2 ovarian cancer with a duration of 5 hours. Recent history of bilateral adnexectomy did not cause gas leakage from the retroperitoneal space. 48 lymph nodes were retrieved, all free from disease. No complications occurred.

Conclusion* We describe here a promising technique that combines all the advantages of the two previously described retroperitoneal accesses without their disadvantages. It can also be extended to any other type of retroperitoneal surgery.

\section{EFFECTIVENESS OF BEVACIZUMAB IN PLATINUM- RESISTANT RECURRENT EPITHELIAL OVARIAN CANCER}

${ }^{1} R$ Pinto*, ${ }^{1} S$ Monteiro, ${ }^{2} B$ Lima, ${ }^{1} A R$ Lopes, ${ }^{1} M$ Abreu, ${ }^{1} M$ Ferreira, ${ }^{1} S$ Sousa, ${ }^{1} P$ Redondo, ${ }^{1} \mathrm{D}$ Pereira, ${ }^{1} \mathrm{~J}$ Sawa-Bordalo. 'Portuguese Oncology Institute of Porto, Portugal; ${ }^{2}$ Hospital Senhora da Oliveira

10.1136/ijgc-2021-ESGO.465

Introduction/Background* bevacizumab is an anti-VEGF monoclonal antibody that has shown efficacy in platinum-resistant recurrent epithelial ovarian cancer (EOC) in clinical trials. Our aim was to evaluate real-world effectiveness of bevacizumab in northern Portugal in this setting.

Methodology retrospective observational series of cases in a single comprehensive cancer center between 2015 and 2020. We reviewed consecutive medical records of platinum-resistant recurrent EOC patients, who underwent bevacizumab with chemotherapy (CT). Primary endpoint was overall survival (OS). Secondary endpoints were overall response rate (ORR), progression free survival (PFS) and safety according to CTCAE v4.0. Descriptive analysis of main demographic, clinical and treatment variables were performed. Kaplan-Meier method was used for OS and PFS.

Result(s)* 21 EOC patients with median age of 61 years (4772 years). Majority were FIGO stage III $(10,47.6 \%)$ or IV $(8,38.1 \%)$ and had high-serous morphology (11, 52.5\%). Most patients had been previously submitted to up-front $(42,9 \%)$ or interval $(42,9 \%)$ debulking surgery. All underwent platinum-based CT as 1st line of treatment (LOT). At baseline, more than half of the patients $(57.4 \%)$ had previously undergone 2 or more LOT. Median number of cycles of bevacizumab was 9 (2-62), concomitant with pegylated liposomal doxorubicin in $10(47.6 \%)$, paclitaxel in $8(38,1 \%)$ and topotecan in $3(14.3 \%)$ patients. Median follow-up time was 19.3 months (4.9-40.8 months). ORR was 42.9\%: 2 (9,5\%) complete response and $7(33.3 \%)$ partial response. Nine patients (42.9\%) had stable disease. Median OS was 25.8 months [IC95\% 11.3-40.3] and PFS 10.8 months [IC95\% 7.0-14.7]. Grade $\geq 3$ hypertension incidence was $14.3 \%$ and grade $\geq 3$ proteinuria $4.8 \%$. There was one death due to gastrointestinal fistula $(4.8 \%)$.

Conclusion* bevacizumab had the predictable safety profile, with better effectiveness than the published in clinical trials, reflecting the small number of patients included in our real- world unicentric series. Nevertheless, new molecular biomarkers and clinical trials urge to improve outcomes in platinum-resistant recurrent ovarian cancer patients in clinical practice.

\section{IS LAPAROSCOPIC INTERVAL DEBULKING SURGERY ACHIEVABLE FOR ADVANCED OVARIAN CANCER AFTER NEOADJUVANT CHEMOTHERAPY?}

M Pellerin*, L Lecointre, C Billard- Martel, T Boisramé, E Faller, T Fabacher, C Akladios. Strasbourg, Strasbourg, France

\subsection{6/ijgc-2021-ESG0.466}

Introduction/Background* Standard treatment of advanced ovarian cancer is complete cytoreductive surgery, historically done by laparotomy, and survival is directly linked to the residual tumor at the end of surgery. When optimal upfront surgery is not achievable, neoadjuvant chemotherapy (NACT) followed by interval debulking surgery (IDS) is an alternative. Laparoscopy as IDS for advanced ovarian cancer is controversial. We wanted to evaluate the feasibility of laparoscopic IDS compared to laparotomy by analyzing overall survival (OS) and progression free survival (PFS), as well as per and postoperative morbidity.

Methodology We conducted a retrospective cohort study and included patients with IDS avec neoadjuvant chemotherapy for advanced ovarian cancer. We included patients with stage III or IV FIGO (International Federation of Gynecology and Obstetrics) serous ovarian cancer who had no residual disease after IDS. We applied a propensity score to match patients on confounding factors.

Result(s)* We included 37 patients in the laparoscopy group and 40 in the laparotomy group, from the $1^{\text {st }}$ of January 2009 to the $1^{\text {st }}$ of June 2019. Median overall survival was 23.1 months (95\% CI 15.7-29.7) and 26.3 months (95\% CI 21.7-31.7) for women who underwent laparoscopy and laparotomy, respectively $(\mathrm{p}=0.17)$. Median PFS was 14.8 months (95\% CI 10.6-21.5) for the laparoscopy group and 12 months (95\% CI 11-15.1) for the laparotomy group $(p=0.057)$. After applying the propensity score, we included 25 patients in each group with similar baseline characteristics. OS was modified with a HR of 0.45 (CI 95\% 0.19-0.95) p $=0.04$ in favor of laparoscopy. Laparotomy group had more early post-operative complications (17 versus 6 in the laparoscopy group, $\mathrm{P}=0.01$ ) and a longer hospitalization time (7.5 days compared to 12.1 days, $\mathrm{p}<0.001)$.

Conclusion* Oncological outcome was better in patients with similar pre-operative characteristics who underwent laparoscopic IDS. Laparoscopic IDS is a safe alternative for laparotomy in selected patients and is associated with less postoperative morbidity.

\section{IMPACT OF SPLENECTOMY DURING INITIAL CYTOREDUCTIVE SURGERY IN ADVANCED STAGE EPITHELIAL OVARIAN CANCER; A NATIONWIDE POPULATION-BASED STUDY}

${ }^{1,2} \mathrm{~S}$ Said*, ${ }^{2} \mathrm{M}$ Van der Aa, ${ }^{1} \mathrm{G}$ Veldmate, ${ }^{1} \mathrm{~J}$ De Hullu, ${ }^{1} \mathrm{~A}$ Van Altena. ${ }^{1}$ Radboud University Medical Center, Deparment of Gynecology and Obstetrics, Nijmegen, Netherlands; ${ }^{2}$ IKNL, locatie Utrecht, Department of Research and Development, Utrecht, Netherlands

10.1136/ijgc-2021-ESGO.467 